\title{
Augusto y el control administrativo y territorial de las zonas mineras del Noroeste hispano
}

\author{
Francisco Javier SÁnChEZ-PALENCIA \\ Consejo Superior de Investigaciones Científicas \\ javier.spalencia@cchs.csic.es \\ Inés SASTRE PRATS \\ Consejo Superior de Investigaciones Científicas \\ ines.sastre@cchs.csic.es \\ Almudea Orejas Saco del Valle \\ Consejo Superior de Investigaciones Científicas \\ almudena.orejas@cchs.csic.es \\ María Ruiz DEL ÁrBoL ${ }^{1}$ \\ Consejo Superior de Investigaciones Científicas \\ maria.ruizdelarbol@cchs.csic.es
}

\section{RESUMEN}

Se presenta una visión de síntesis sobre las reformas de Augusto en el Noroeste hispano y el impacto que supusieron sobre la organización social y territorial. Esta reorganización fue la base sobre la que se asentó la puesta en marcha de la minería de oro, que debe considerarse un elemento más dentro de la explotación integral de los recursos provinciales, no como una actividad económica sectorial. La síntesis se apoya en los resultados de intervenciones recientes en diversas zonas mineras del Noroeste peninsular, entre los que se destacan aquellos que responden a medidas tomadas en época de Augusto o que son consecuencia directa de ellas.

Palabras clave: Minería antigua. Administración provincial. Tributación. Campesinado. Alto Imperio romano. Hispania. Noroeste de la Península Ibérica.

\section{Augustus and the Administrative and Territorial Control of Mining Zones of Northwestern Hispania}

\begin{abstract}
An overall picture about the reforms of Augustus in the Hispanic Northwest and assumed impact on social and territorial organization is presented. This reorganization was the basis for the implementation of gold mining, to be considered as one element in the comprehensive exploitation of provincial resources, not as a sectoral economic activity. The synthesis is based on the results of recent interventions in

\footnotetext{
1 Grupo de investigación Estructura social y territorio. Arqueología del paisaje (IH, CCHS, CSIC). Este trabajo se inserta en el proyecto de investigación "Paisajes de dominación y resistencia. Procesos de apropiación y control social y territorial en el Noroeste hispano" (PADORE, HAR2012-33774), financiado por el Ministerio de Economía y Competitividad.
} 
various mining areas of peninsular Northwest, among which stand out those that respond to measures taken in time of Augustus or are a direct result of them.

KeyWords: Ancient Mining. Provincial administration. Taxation. Peasants. Early Roman Empire. Hispania. Northwest of the Iberian Peninsula.

Sumario: 1. El impacto de la conquista romana: la implantación administrativa (civitates y suelo público). 2. Las bases administrativas, fiscales y jurídicas de la explotación de las minas: la mano de obra local y el control imperial.

Este trabajo es una síntesis de las aportaciones sobre el tema que ha hecho el grupo de investigación "Estructura social y territorio - Arqueología del paisaje" del IH (CCHS, CSIC) a lo largo de las últimas tres décadas en el Noroeste y Occidente peninsulares. Los resultados aportados tras todos estos años de trabajo suponen una renovación de la perspectiva que se ha venido aplicando al estudio de los territorios provinciales en Occidente, y proporcionan una visión diferente del proceso de integración de estos territorios en el Imperio. En gran parte de las zonas estudiadas la minería del oro antigua tuvo un especial desarrollo, de modo que esa actividad fue un factor muy relevante de todos estos procesos, pero su impacto debe analizarse dentro de las formas de relación social y territorial que se ponen en marcha tras la conquista. Si bien es evidente que existe una administración minera, con procuratores metallorum, metalla etc., partimos de la base de que la explotación minera no responde a una sectorialización ni económica ni administrativa ni territorial.

\section{El impacto de la conquista romana: la implantación administrativa (civitates y suelo público)}

A la hora de valorar la incidencia que tuvo la conquista romana del Noroeste, el primer parámetro con que ha de contarse es saber cuáles fueron y hasta donde llegaron las formas de ocupación y explotación del territorio en época prerromana. Esta cuestión es más importante aun cuando se trata de la minería aurífera. Siempre hemos defendido que sólo se explotaron los placeres fluviales, los no consolidados, los que arrastran los ríos. Pero, precisamente por esa movilidad de los placeres, resulta muy difícil documentar la evidencia arqueológica directa de tal hecho. Sin embargo, recientemente y de forma muy afortunada esa dificultad ha sido superada. En una construcción de los siglos IV-III a.C. del castro prerromano de La Ciguadeña, situado al borde del río Duero en la zona minera de Pino del Oro, se documentó una partícula o pepita de oro dentro de su nivel de ocupación. ${ }^{2}$ Se daba la circunstancia de que, además, durante la realización de unas prospecciones a la batea previas se habían localizado ya placeres auríferos en las inmediaciones del asentamiento, a unos $100 \mathrm{~m}$

2 SÁncheZ-Palencia ET ALII 2013. 
de él, en el cauce del arroyo de Fuentelarraya y de que la partícula encontrada en el poblado era en todo semejante por su morfología a las de ese placer: formas globulares con todo su perímetro claramente redondeado por la acción erosiva del transporte sufrido. Esto quiere decir que, por primera vez en todo el Noroeste, contamos ya con una evidencia arqueológica dentro de un contexto cerrado y bien datado de la práctica del bateo por las comunidades prerromanas, algo que antes sólo podíamos apoyar en datos arqueológicos indirectos, en esencia la producción orfebre, y en las fuentes literarias antiguas.

Las primeras dataciones útiles para documentar el inicio de la minería del oro siguen siendo las procedentes de asentamientos directamente relacionados con las minas de la Valduerna. Esta región pudo ser de las primeras en ser explotadas, dada su cercanía a Asturica Augusta, centro administrativo del que seguramente acabaron dependiendo todas las explotaciones del Noroeste. ${ }^{3}$ Las fechas se corresponden con el gobierno de Tiberio. ${ }^{4}$ Más recientemente también el Occidente de Asturias ha aportado fechas tempranas para las explotaciones ${ }^{5}$-aunque no podemos admitir una supuesta datación prerromana-. De todos modos, la puesta en marcha de las minas implicaba un trabajo previo de localización de los principales yacimientos, de prospección sistemática y de preparación de toda la infraestructura que debió de haberse iniciado poco después de la conquista. Este trabajo preparatorio, que ya había sido puesto de relieve claramente en algunas zonas de Asturias ${ }^{6} \mathrm{o}$, más recientemente en Las Cavenes de El Cabaco, ${ }^{7}$ se ha vuelto a evidenciar mediante trabajos de arqueología experimental en la zona minera de Pino del Oro. ${ }^{8}$ Lo más relevante en este punto es destacar que la explotación sistemática del oro desde, al menos, el 15 d.C. no habría sido posible sin la profunda reorganización social y territorial que impulsó el gobierno de Augusto en todo el Noroeste desde el momento mismo de la conquista.

En la actualidad es recurrente la idea de que el siglo I d.C., hasta la época flavia, es un periodo continuista en el cuál perviven las tradiciones indígenas con una mínima interferencia romana, la indispensable para mantener el orden tras la guerra. Esto se traduce en una supuesta inexistencia de cambios estructurales, de tal manera que el impacto de la dominación no se hace visible prácticamente hasta el siglo II. Suponen algunos autores que la organización social y territorial castreña era perfectamente compatible con las primeras formas de dominación romana; que Roma adoptó las estructuras étnico-políticas indígenas como base de su sistema imperial provocando, incluso, un "florecimiento" cultural dentro de una fase incluida como "castreñoromana" en las periodizaciones sobre la Edad del Hierro. Así, suele retrotraerse hasta el siglo II la existencia de auténticas civitates, puesto que previamente son los populi indígenas la base de la administración ${ }^{9}$ dentro de marcos territoriales amplios como

3 Orejas - Morillo 2013.

4 Domergue - Sillières 1977.

5 Villa 2010; Rozas - CABO 2002.

6 SÁnCheZ-PALENCIA - SuÁREZ 1985.

7 Sánchez-Palencia et alit 2006; Sánchez-Palencia 2011.

8 SÁnchez-Palencia et alit 2013.

9 Alarcîo 1995-6. 
los conventus, necesarios para contrarrestar la fragmentación, dispersión y ruralidad del poblamiento. ${ }^{10}$

No es posible defender una estructura territorial basada en populi en época prerromana, que pueda ser reutilizada por Roma. No vamos a entrar ahora a argumentar esta idea, que se deduce claramente de las investigaciones sobre la Edad de Hierro que hemos desarrollado en numerosas publicaciones, ${ }^{11}$ así como de los trabajos de otros investigadores. ${ }^{12}$ Pero, al margen de esto, la civitas romana está documentada en el Noroeste en general desde los primeros momentos, y muy claramente a lo largo del siglo I d.C. Así lo indica el Edicto de El Bierzo del 15 a.C., ${ }^{13}$ documento sobre el que volveremos más adelante y que nos permite afirmar que en los territorios de El Bierzo y Valdeorras mencionados en él estaba ya operativa una estructura de civitates con funciones administrativas, políticas y fiscales en el año 15 a.C. Para otras regiones en estos momentos tempranos tenemos también documentación epigráfica sobre la existencia de civitates. ${ }^{14}$

Las medidas de Augusto implicaron, por lo tanto, una intervención directa sobre territorios y poblaciones que dio lugar a la implantación de un sistema administrativo homogéneo para todo el Noroeste. Las diferencias que presenta esta organización a escala local y regional están condicionadas por los intereses concretos del poder imperial, no tanto por la realidad indígena sobre la que se actuaba. La civitas es la estructura administrativa impuesta por el imperialismo romano en un contexto marcado por el derecho de conquista, y no por la tradición prerromana. De la lectura del De agrorum qualitate de Frontino se desprende que el modelo de civitates peregrinae era característico del suelo tributario en época de Augusto. ${ }^{15}$ Se trata de un tipo de organización territorial que encaja muy bien con las formas de dominación imperial que caracterizan los inicios del Principado. En este momento, como ya indicó en su día Nicolet, ${ }^{16}$ resultaba esencial sistematizar los mecanismos de control y explotación del Imperio para garantizar el flujo de recursos desde las provincias y superar las limitaciones de la "economía de guerra" que caracterizaba la expansión republicana. ${ }^{17} \mathrm{Al}$ mismo tiempo cada vez se recurrió más al sometimiento peregrino frente a la esclavización como elemento básico para consolidar el control territorial y la tributación regular. ${ }^{18}$

Las comunidades peregrinas se configuraban tras la deditio, cuando se respetaba la libertad de los sometidos y se reconocía el control sobre sus territorios a cambio de una tributación sobre el suelo. Ese territorio era definido de acuerdo con los inte-

10 García Fernández 1996, 60.

11 Fernández-Posse - Sánchez-Palencia 1988; Orejas 1996; Fernández-Posse 1998; FernándezPosse - Sánchez-Palencia 1998; Sastre 2002 y 2008.

12 Vid. Sastre 2009; Esparza 2010.

13 SÁnchez- Palencia - Mangas 2000.

14 Civitas Lougeiorum en el 1 d.C. (BALBín 2006, $201 \mathrm{n}^{\circ} 49$ ); hitos terminales leoneses IRPL 304-312; numerosas inscripciones datables en el siglo I d.C. con menciones de origo.

15 Th. 1-2; Orejas - SaStre 1999.

16 Nicolet 1988.

17 NACO 2003.

18 Gonzales 2002; Plácido 2008. 
reses romanos y era puesto bajo el control de gobiernos locales conformados por los grupos y familias que resultaban más convenientes para el Estado. Contrariamente a las tesis patrimonialistas que inauguró Mommsen, consideramos que este suelo no es ager publicus, sino ager provincialis o ager peregrinus, perfectamente susceptible de ser propiedad privada pleno iure, si bien no optimo iure. ${ }^{19}$ Sin embargo, es posible que en un primer momento estas comunidades tributaran en bloque. Fueron, por todo ello, los sujetos fiscales básicos del ordenamiento tributario. De ahí la necesidad de delimitar claramente el territorio de cada civitas, de adscribir a cada una sus correspondientes poblaciones y de reseñar convenientemente todo esto en el censo provincial. ${ }^{20}$ Este proceso temprano se ha podido documentar en el Noroeste fundamentalmente a partir de la información epigráfica.

En el Edicto de El Bierzo, del 15 a.C., el término civitas aparece como tal, y con el sinónimo gens, para indicar las demarcaciones administrativas sujetas a tributación. Este término gens aparece en la documentación de otras regiones del Imperio, como en los Alpes, en los que se remata la conquista también en este momento conforme a criterios de dominación muy semejantes. ${ }^{21}$ El Edicto, como es sabido, da cuenta de la concesión de inmunidad tributaria a una comunidad local (castellani) y pone de manifiesto la centralidad de la fiscalidad en la ordenación poblacional y territorial de la zona. La concesión del privilegio (primera parte del texto) implica una ulterior intervención (segunda parte del texto), readscribiendo poblaciones para compensar y equilibrar las cargas fiscales..$^{22} \mathrm{Al}$ mismo tiempo, es evidente otro plano de intervención imperialista, claramente político: premiar a los leales, y someter a los rebeldes. Es decir, el Edicto refleja los dos ejes esenciales de la dominación: la construcción de una clase dominante privilegiada, y la sistematización de la explotación del territorio a través del sistema tributario.

Existe, por otro lado una clara plasmación sobre el territorio de esas realidades descritas por el Edicto. Las investigaciones llevadas a cabo en particular en la zona arqueológica de Las Médulas ${ }^{23}$ han permitido identificar e interpretar un modelo de poblamiento romano que nada tiene que ver con el prerromano. Ese cambio de modelo ya había sido identificado de forma general en Corporales y en las cuencas del Ería y el Cabrera, ${ }^{24}$ y también más tarde y en la cuenca noroccidental del Duero. ${ }^{25}$ Pero en el caso de Las Médulas se pueden establecer unas tendencias en la actividad principal de los diversos asentamientos que son complementarias entre sí y que indican sobre todo una explotación integral y sistemática de los recursos, sin duda controlables tributariamente, y que permitiría a su vez una redistribución de los mismos. El papel primordial de las clases dominantes se llega a manifestar en diversos elementos y estructuras del registro arqueológico, por ejemplo si se compara el de Las Pedreiras de Lago, con una implantación en forma de domus plenamente romana

19 Orejas - Sastre 1999; Sastre 2002, 97 ss.

20 Orejas - Sastre 1999; France 2001.

21 Laffi 1966; LuRASChi 1979; VAlvo 2012; SAStre - Orejas e.p.

22 OREJAS ET ALII 2000.

23 SÁNCHEZ-PALENCIA 2000.

24 Fernández-Posse - SÁnchez-Palencia 1988.

25 Orejas 1996. 
y toda una serie de "nuevos materiales" romanos, con el del poblado metalúrgico de Orellán, que albergaba a una mano de obra no cualificada y cuyo registro material es menos diversificado, más "pobre". En otras zonas, como es el caso de Pino del Oro, ${ }^{26}$ esa presencia de las clases dominantes se puede seguir mejor a través de un registro epigráfico no menos emulante y diferenciador.

El resultado de todo esto es la aparición de una sociedad provincial, tributaria y campesina, en el sentido sociológico del término, que tiene su reflejo en una nueva configuración del paisaje, jerarquizado y rural. Este no es herencia del mundo prerromano, como ya se ha indicado, pero tampoco responde al modelo idealizado que ha sido forjado por la tradición historiográfica de la "romanización". No es un territorio de ciudades y esclavos. Es un mundo marcadamente rural y campesino que sólo es comprensible dentro de las formas de dominación imperial augusteas. Desde el punto de vista arqueológico se documenta un registro que parcialmente puede tener una impronta mayor o menor indígena, pero que es claramente diferente del prerromano en cuanto a patrones de poblamiento, diversificación de la morfología de los poblados (los elementos que recuerdan lo castreño son elementos aislados), modificación de los ajuares domésticos y las técnicas constructivas e introducción de nuevas prácticas culturales como, por ejemplo, el uso de la escritura epigráfica.

\section{Las bases administrativas, fiscales y jurídicas de la explotación de las minas: la mano de obra local y el control imperial}

En un contexto marcado por la imposición de una dominación peregrina, el Estado romano se reservó la propiedad de las explotaciones mineras auríferas. El carácter público de las minas es algo admitido hace tiempo tanto por historiadores y arqueólogos, ${ }^{27}$ como juristas, ${ }^{28}$ y se deriva directamente de las fuentes. Así lo menciona explícitamente Estrabón (Str., III 2.10). También se deduce del texto de Dión Casio (D.C., LII 28.4) en el que Mecenas aconseja a Augusto sistematizar los ingresos provenientes de recursos públicos, entre ellos, las minas. Hay que tener en cuenta, además, que el oro era un bien estratégico para el Estado, en el marco de la reforma monetaria impulsada por el propio Augusto. ${ }^{29}$

Por otra parte, el tipo de minería y el carácter de las propias explotaciones sólo eran posibles por medio del control estatal. El alcance de los frentes de explotación $\mathrm{y}$, sobre todo, de la red hidráulica supone un control suprarregional que sólo es asumible para el Estado. En este sentido, es interesante resaltar la diversa morfología respecto de las labores mineras de la Bessa, ${ }^{30}$ precedentes sin duda a la hispanas en la explotación aurífera, y que, al contrario de las del Noroeste peninsular, se hicieron por un sistema de concesiones que controlaban publicani, como indica claramente

\footnotetext{
26 SASTRE ET ALII 2010.

27 Domergue 1990, 295 ss; Hirt 2010.

28 MATEO 2001, 176 ss.

29 Crawford 1982; Lo Cascio 1981; Corbier 1989; Sánchez-Palencia 2000.

30 SÁnChez-Palencia ET ALII 2011.
} 
Plinio (Plin., NH 33.80). Al mismo tiempo, el carácter extensivo, móvil y disperso de las explotaciones hace inviable un modelo de explotación basado en "distritos", al menos en el sentido de Vipasca. Dada la amplia extensión del territorio con minas en el Noroeste hispano, es posible pensar que las distintas zonas mineras tuvieron su propia denominación y que a partir de cierto momento avanzado existieron varios procuratores metallorum que se ocuparon de diferentes regiones. ${ }^{31}$ Pero es improbable que existieran "distritos" en el sentido administrativo, ni mucho menos arrendamientos de las explotaciones a privados.

Lo que sí es necesario suponer es que la distinción entre el ager publicus minero y el suelo peregrino estaba muy clara. Siguiendo el trabajo de algunos juristas, principalmente el trabajo clásico de Negri ${ }^{32}$ y teniendo en cuenta también las visiones de otros autores ${ }^{33}$ podemos afirmar que existía desde bastante temprano una distinción entre el derecho sobre el suelo y sobre el subsuelo, unida a una separación y coexistencia entre bienes agrarios y mineros. Así mismo, es fundamental el hecho de que la propiedad se reconocía a quien ejercía la explotación. Todo esto permite pensar que probablemente tras la conquista y la definición de comunidades peregrinas, quedó reservada al fisco la propiedad del subsuelo y el derecho de explotación del mismo. Esta propiedad se fue haciendo efectiva conforme avanzó el proceso de explotación y se fue evidenciando en el propio territorio minero. ${ }^{34}$

A partir de estos presupuestos, puede afirmarse que el éxito de la explotación minera del Noroeste radicó en la imbricación entre administración imperial y administración local. El suelo minero, como res fiscalis, era administrado directamente por el Estado. Probablemente en este primer momento, el papel del ejército fuera fundamental. Las técnicas extractivas a gran escala, cuyo precedente directo más próximo está en la minería republicana del norte de Italia, ${ }^{35}$ seguramente fueron implantadas con el apoyo técnico de personal militar, que habría contribuido a la ordenación del territorio y con ello al inicio de las explotaciones y al trazado de la infraestructura hidráulica asociada a ellas. ${ }^{36}$ En varias zonas del cuadrante noroccidental puede rastrearse un testimonio de esta presencia militar dentro de las zonas mineras explotadas en los primeros momentos. Tal sería el caso de dos recintos campamentales cuya ocupación fue muy reducida en el tiempo y que fueron destruidos claramente por las operaciones de puesta en explotación de sendos yacimientos auríferos. El primero en ser descubierto fue el campamento de Valdemeda (Manzaneda, León), en la cuenca del río Ería, que no cuenta con ningún elemento de datación absoluta, pero que seguramente habría que colocar en la primera mitad del siglo I d.C. por el contexto

31 Procuratores Augusti y metallorum en la Valduerna, RABANAL - GARCía 2001, n64-68, 70-72 y, en un caso, la mención del nombre del metallum (Ulpius Eutyches, proc. metall. Alboc. CIL II, 2598, Chaves). Esta referencia se ha hecho concordar con la mención de Plinio de un metallum en Gallaecia (NH 33.80: metallo [...] Albucrarense).

32 NEGRI 1985.

33 Domergue 1990, 229-240; Hirt 2010, 82-93.

34 SASTRE 2012; OREJAS ET ALII 2012.

35 SÁnchez-Palencia ET ALII 2011.

36 SAStre - SÁnCheZ-PALEnCia 2002; SASTre et aliI 2010. 
general del entorno en el que se sitúa. ${ }^{37}$ A partir del cambio de Era se situaría la ocupación del segundo campamento, el destruido por las labores de la Mina da Presa en la zona minera de Penamacor-Meimoa, ${ }^{38}$ para el que se cuenta con la fecha post quem de un tesorillo de denarios cuya ocultación se fecha en el 14-2 a.C. También hacia esos momentos tempranos es patente la presencia del ejército en los ejes de control esencial del territorio: los campamentos militares que ya a partir de Tiberio darán paso a entidades urbanas. ${ }^{39} \mathrm{Al}$ mismo tiempo, la definición de las civitates y la imposición del sistema tributario hicieron posible el suministro de mano de obra. Como ya hemos defendido y argumentado en otras ocasiones, entre las cargas fiscales estaba la obligación de trabajar en las minas que recaía sobre la población campesina de las civitates..$^{40}$ De hecho, Floro (II 33) también explicita la explotación directa de las minas astures con mano de obra local bajo Augusto.

Esta división administrativa y jurídica del suelo minero no debe confundirse como una sectorialización de esta actividad. De lo dicho hasta ahora se puede deducir exactamente lo contrario: la minería forma parte de una explotación sistemática e integral de los recursos imperiales, y participa del mundo rural de una manera claramente ventajosa para los intereses imperialistas. La afirmación de que el Noroeste romano fue un ámbito rural y campesino no se limita a los aspectos morfológicos del registro. Tiene unas implicaciones históricas de primera magnitud. ${ }^{41}$ Las formas productivas campesinas son ajenas a la especialización productiva y a la maximización de beneficios, y se caracterizan por: autosuficiencia, trabajo familiar, búsqueda de la reproducción simple de la unidad doméstica, prevención de la sobre-explotación del trabajo -no se produce más allá de lo que culturalmente fijado como "digno"- diversificación de la producción, valores de uso... La explotación imperialista del trabajo campesino resulta muy eficaz en determinados contextos provinciales, porque permite la externalización de los costes de la mano de obra. Una característica fundamental del campesinado es la tendencia a la subproducción arriba indicada y la existencia de un subempleo estacional. Esto permite incluir en la esfera doméstica actividades complementarias a la explotación agraria, como la práctica artesanal, o trabajar estacionalmente fuera del ámbito doméstico, ya sea como asalariados en grandes explotaciones agrarias (como en Italia) o con prestación de jornadas laborales para el trabajo minero (o cualquier obra pública exigida por el Estado).

Siguiendo a Erdkamp "the economy of the ancient world was in most regions not sufficiently developed to sustain a large non-agricultural population without the element of externalization of reproductive cost, which provided a significant pool of labour for the non-agricultural sectors. While undoubtely the rural population almost exclusively consisted of peasant and small farmers, whether tenants or not, and of commercial farmers and their slaves and day-labourers, this did not preclude a significant element of non-agricultural labour in transport and manufacture, which was

\footnotetext{
37 SÁNChez-PALENCia 1986.

38 SÁNCHEZ-PALENCIA - PÉREZ 2005.

39 Morillo 2006; Orejas - Morillo 2013.

40 Mangas - Orejas 1999; Orejas - Sastre 1999; Sánchez-Palencia 2000.

41 Wolf 1966; Shanin 1971; SeVilla - PÉrez 1976.
} 
economically viable because it was cheap. These sectors, however, remained inextricably connected to agriculture; by its nature, such non-agricultural labour could not exist disconnected from agriculture. Only in those places where the demand for labour from the non-agricultural sector was sufficiently high and stable could a large element of labour exist independently from agriculture". ${ }^{42} \mathrm{Y}$ este no es el caso de la minería del Noroeste, a pesar de la envergadura de las explotaciones.

Este esquema campesino es, a nuestro juicio, contrario a la "sectorialización productiva": la explotación minera exige una reorganización completa de toda la actividad económica y no puede desarrollarse al margen de la actividad agraria. El tipo de trabajo que exigía la minera aurífera del Noroeste no implicaba una especialización ni una dedicación que justifique la idea de una mano de obra incompatible con el mundo agrario. Todo lo contrario. Esto es claramente visible en el registro arqueológico de las zonas con minas: la principal diferenciación "funcional" de los asentamientos tiene que ver con la jerarquización social, y no con una sectorialización económica. Las actividades artesanales especializadas -como puede ser el caso de la metalurgia de hierro en el asentamiento de Orellán, en Las Médulas (León), o la producción textil de Fuente de la Mora, en El Cabaco (Salamanca) - ${ }^{43}$ se documentan dentro de asentamientos con una clara vocación agraria. Estamos ante comunidades campesinas que trabajan a tiempo parcial en actividades no agrarias, entre ellas estacionalmente el trabajo minero, y cotidianamente la vigilancia y mantenimiento de los canales.

\section{BibLiografíA}

Alarcîo, J. (1995-6): “As civitates do Norte de Portugal”, Cadernos de Arqueologia II série $12 / 13,25-30$.

Corbier, M. (1989): "Histoire monétaire, histoire des prix, histoires des mines", [en] C. Domergue (coord.), Minería y metalurgia en las antiguas civilizaciones mediterráneas y europeas II, Madrid, 183-194.

Crawford, M. H. (1982): La moneta in Grecia e a Roma, Bari.

ERDKAMP, P. (1999): “Agriculture, underemployment and the cost of rural labour in the Roman world", Classical Quarterly 49/2, 556-572.

Esparza, A. (2010): "Etnicidad y arqueología en Asturia", [en] P. Bueno - A. Gilman, C. Martín - F. J. Sánchez-Palencia (eds.), Arqueología, sociedad, territorio y paisaje. Estudios sobre Prehistoria Reciente, Protohistoria y transición al mundo romano en Homenaje a $M^{a}$ Dolores Fernández-Posse (=Bibliotheca Praehistorica Hispana XXVIII), Madrid, 291310.

FERNÁNDEZ-Posse, M. D. (1998): "Las comunidades campesinas en la cultura castreña", Trabajos de Prehistoria 55/2, 127-150.

Fernández-Posse, M. D. - SÁnchez-Palencia, F. J. (1988): La Corona y el Castro de Corporales II, Madrid.

42 ERDKAMP 1999, 572.

43 SÁnchez-Palencia 2000: 364 ss; SáncheZ-Palencia - Ruiz del Árbol 1999. 
FrANCE, J. (2001): "Remarques sur les tributa dans les provinces nord-occidentales du HautEmpire romain (Bretagne, Gaules, Germanies)", Latomus 60/2, 359-79.

García Fernández, E. (1996): "El desarrollo de la municipalización latina: la Bética y el Noroeste", [en] S. Reboreda - P. López-Barja (eds.), A cidade e o mundo: romanización e cambio social, Xinzo de Limia, 147-164.

Gonzales, A. (2002): "Provenance des esclaves au Haut Empire. Pax romana et approvisionnement”. Routes et marchés d'esclaves. 26 colloque du GIREA, Besançon, 65-85.

HiRT, A. M. (2010): Imperial mines and quarries in the Roman World. Organizational aspects $27 B C-A D$ 235, Oxford.

Laffi, U. (1966): Adtributio e contributio. Problemi del sistema politico-amministrativo dello Stato romano, Pisa.

Lo CASCIO, E. (1981): "State and coinage in the Late Republic and Early Empire", Journal of Roman Studies 71, 76-86.

LuRAsCHI, G. (1979): Foedus, ius Latii, civitas. Aspetti costituzionali della romanizzazione in Transpadana, Padova.

Mangas, J. - Orejas, A. (1999): "El trabajo en las minas en la Hispania Romana”, [en] J. F. Rodríguez-Neila - C. González-Román - J. Mangas - A. Orejas (eds.), El trabajo en la Hispania romana, Madrid, 207-337.

Mateo, A. 2001: Observaciones sobre el régimen jurídico de la minería en tierras públicas en época romana, Santiago de Compostela.

Morillo, A. (2006): "Roman Army and urban development in the northwest Spain: Asturica Augusta and Legio VII Gemina", [en] L. Abad - S. Keay - S. Ramallo (eds.), Early Roman Towns in Hispania Tarraconense (IInd. C. B. C.-Ist C. A.D.) (=Journal of Roman Archaeology Supplementary series 62), 197-211.

NeGRI G. (1985): Diritto minerario romano I. Studi esegetici sul regime delle cave private nel pensiero dei giuristi classici, Milano.

Nicolet, C. (1988): L'inventaire du monde. Géographie et politique aux origines de l'Empire romaine, Paris.

ÑACO, T. (2003): Vectigal incertum. Economía de guerra y fiscalidad republicana en el occidente romano: su impacto histórico en el territorio (218-133 a. C.) (=BAR International Series 1158), Oxford.

OREJAS, A. (1996): Estructura social y territorio. El impacto romano en la cuenca nordoccidental del Duero (=Anejos de Archivo Español de Arqueología XV), Madrid.

Orejas, A. - Morillo, A. (2013): “Asturica Augusta. Reflexiones sobre su estatuto y su papel territorial (finales del siglo I a.C.- principios del siglo III d.C.)”, [en] R. Cid - E. García (eds.), Debita verba. Estudios en homenaje al profesor Julio Mangas Manjarrés, Oviedo, 93-120.

Orejas, A. - SAStre, I. (1999): "Fiscalité et organisation tu territoire dans le Nord-Ouest de la Péninsule Ibérique: civitates, tribut et ager mensura conprehensus", Dialogues d'Histoire Ancienne 25/1, 159-188.

Orejas, A. - Sastre, I. - Sánchez-Palencia, F. J. - Plácido, D. (2000): "El edicto de Augusto de El Bierzo y la primera organización romana del Noroeste peninsular", [en] F. J. Sánchez-Palencia - J. Mangas (eds.), El Edicto de El Bierzo. Augusto y el Noroeste de Hispania, Ponferrada, 63-112. 
Orejas, A. - Sastre, I. - Zubiaurre, E. (2012): “Organización y regulación de la actividad minera hispana altoimperial", [en] M. Zarzalejos - P. Hevia - L. Mansilla (eds.), Paisajes mineros antiguos en la Península Ibérica. Investigaciones recientes y nuevas líneas de trabajo. Homenaje a Claude Domergue, Madrid, 31-46.

Plácido, D. (2008): "La théorie de l'égalité des êtres humains et l'évolution des formes de dépendance", [en] La fin du statut servile? Affranchisement, libération, abolition. XXX colloque du GIREA, Besançon, 467-473.

RozAs, V. - CABO, L. (2002): “Dataciones geoquímicas y dendrocronológicas de época romana en Asturias", [en] M. A. Blas - A. Villa (eds.), Los poblados fortificados del noroeste de la Península Ibérica: formación y desarrollo de la cultura castreña: Coloquios de Arqueología en la cuenca del Navia, Navia, 345-356.

SÁncheZ-PALENCIA, F. J.

(1986): "El campamento romano de Valdemeda, Manzaneda (León): ocupación militar y explotación aurífera en el NW peninsular", Numantia 2, 227-234.

(2011): "Minería romana en el Noroeste de Hispania: tecnología minera y explotación del territorio", [en] C. Braz - A. M. S. Bettencourt - J. I. F. P. Martins - J. Carvalho (coords), Povoamento e exploraçaô dos recursos mineiros na Europa atlântica ocidental, Braga, 113-131.

Sánchez-Palencia F. J. (ed.) (2000): Las Médulas (León). Un paisaje cultural en la Asturia Augustana, León.

SÁnchez-Palencia, F. J. - Orejas, A. - Sastre, I. - Pérez, L. C. (2006): "Las zonas mineras romanas del Noroeste peninsular: infraestructura y organización del territorio", [en] Nuevos elementos de ingeniería romana. III congreso de las obras públicas romanas, Astorga, 265-285.

SÁnchez-Palencia, F. J. - Pérez, L. C. (2006): "Mineria romana de oro en las cuencas de los ríos Erges/Erjas y Bazágueda: la zona minera de Panamacor-Meimoa", [en] Lusitanos e Romanos no Nordeste da Lusitania, Guarda, 267-307.

SÁnchez-Palencia, F. J. - Romero, D. - Beltrán, A. (2013): "Paisajes mineros en el Noreste de Lusitania y Asturia meridional", [en] M. Zarzalejos - P. Hevia - L. Mansilla (eds.): Paisajes mineros antiguos de la Península Ibérica. Investigaciones recientes y nuevas líneas de trabajo. Homenaje a Claude Domergue, Madrid, 155-170.

SÁnchez-Palencia, F. J. - Ruiz del Arbol, M. (1999): "La minería aurífera romana en el Nordeste de Lusitania: Las Cavenes de El Cabaco (Salamanca)", Archivo Español de Arqueología 72, 179-180, 119-140.

SÁnchez-Palencia, F. J. - SuÁrez SuÁrez, V. (1985): "La minería antigua de oro en Asturias", [en] Asturias. Libro de la Mina, Vitoria, 221-241.

Sánchez-Palencia, F. J. - Vaudagna, A. - Pecharromán, J. L. - Beltrán, A. - Currás, B. - Alonso, F. - Ruiz del Árbol, M. (2011): "La zona minera de la Bessa (Biella, Italia) como precedente republicano de la minería de oro en Hispania", [en] P. Bueno - A. Gilman - C. Martín - F.J. Sánchez-Palencia (eds.), Arqueología, sociedad, territorio y paisaje. Estudios sobre Prehistoria Reciente, Protohistoria y transición al mundo romano en homenaje a M. Dolores Fernández-Posse, (=Bibliotheca Praehistorica Hispana XXVIII), Madrid, 329-347. 
SASTRE, I.

(2002): "Forms of social inequality in the Castro Culture of Northwest Iberia", European Journal of Archaeology 5/2, 213-48.

(2008): "Community, identity and conflict. Iron age warfare in the Iberian Northwest", Current Anthropology 49/6, 1021-36.

(2012): "Las zonas mineras auríferas en el sistema provincial altoimperial: el caso del Noroeste hispano", [en] A. Orejas - C. Rico (eds.), Minería y metalurgia antiguas. Visiones y revisiones. Homenaje a Claude Domergue (=Collection de la Casa de Velázquez 128), Madrid, 255-259.

SASTRE, I. (coord.) (2009): Arqueología espacial: Identidades. Homenaje a $M^{a}$. Dolores Fernández-Posse, (=Arqueología Espacial 27), Teruel.

Sastre, I. - Beltrán, A. - SÁnchez-Palencia, F. J. (2010): “Ejército y comunidades locales en el Noroeste peninsular: formas de control y relaciones de poder en torno a la minería del oro", [en] J. J. Palao (ed.), Militares y civiles en la antigua Roma. Dos mundos diferentes, dos mundos unidos, Salamanca, 117-134.

SAStre, I. - Orejas, A. (e.p.): "Pervivencia y cambio en el proceso de dominación romana del Occidente de Hispania", [en] Actas del XXXVI coloquio del GIREA, Barcelona.

SAStre, I - SÁnchez-PALEnCiA, F. J. (2002): "La red hidráulica minera del Noroeste: aspectos jurídicos, administrativos y políticos", Archivo Español de Arqueología 75, 215-234.

Sevilla, E. - PÉREz, M. (1976): "Para una definición sociológica del campesinado", Agricultura y sociedad 1, 15-39.

Shanin, T. (1971): Peasants and Peasant Societies: Selected Readings, Baltimore.

VAlvo, A. (2012): “Gentes Alpinae sub imperium p. R. redactae. I postumi di una sconfitta”, [en] F. Marco - F. Pina - J. Remesal (eds.), Vae victis! Perdedores en el mundo antiguo, Barcelona, 153-159.

Villa, A. (2010): "El oro en la Asturias Antigua: beneficio y manipulación de los metales preciosos en torno al cambio de era”, [en] J. A. Fernández-Tresguerres (coord.), Cobre y oro: minería y metalurgia en la Asturias prehistórica y antigua, Oviedo, 83-126.

Wolf, E. (1966): Peasants, Prentice-Hall. 